\title{
A Genome-Wide Association Study of Age-Related Hearing Impairment in Middle- and Old-Aged Chinese Twins
}

\author{
Haiping Duan $\mathbb{D}^{1,2,3}$ Wanxue Song $\mathbb{D}^{1},{ }^{1}$ Weijing Wang $\mathbb{D},{ }^{1}$ Hainan Cao ${ }^{1}{ }^{4}$

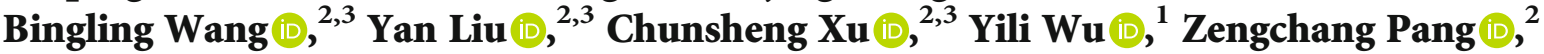 \\ and Dongfeng Zhang $\oplus^{1}$ \\ ${ }^{1}$ Department of Epidemiology and Health Statistics, Public Health College, Qingdao University, No. 38 Dengzhou Road, \\ Shibei District, Qingdao, 266021 Shandong Province, China \\ ${ }^{2}$ Qingdao Municipal Center for Disease Control and Prevention, No. 175 Shandong Road, Shibei District, Qingdao, \\ 266033 Shandong Province, China \\ ${ }^{3}$ Qingdao Institute of Preventive Medicine, No. 175 Shandong Road, Shibei District, Qingdao, 266033 Shandong Province, China \\ ${ }^{4}$ Department of Otorhinolaryngology, Qingdao Municipal Hospital, Qingdao, 266011 Shandong Province, China
}

Correspondence should be addressed to Dongfeng Zhang; zhangdf1961@126.com

Received 23 July 2020; Revised 17 June 2021; Accepted 3 July 2021; Published 19 July 2021

Academic Editor: Cheol Lee

Copyright (C) 2021 Haiping Duan et al. This is an open access article distributed under the Creative Commons Attribution License, which permits unrestricted use, distribution, and reproduction in any medium, provided the original work is properly cited.

\begin{abstract}
Background. Age-related hearing impairment (ARHI) is considered an unpreventable disorder. We aimed to detect specific genetic variants that are potentially related to ARHI via genome-wide association study (GWAS). Methods. A sample of 131 dizygotic twins was genotyped for single-nucleotide polymorphism- (SNP-) based GWAS. Gene-based test was performed using VEGAS2. Pathway enrichment analysis was conducted by PASCAL. Results. The twins are with a median age of 49 years, of which 128 were females and 134 were males. rs6633657 was the only SNP that reached the genome-wide significance level for better ear hearing level $(\mathrm{BEHL})$ at $2.0 \mathrm{kHz}\left(P=1.19 \times 10^{-8}\right)$. Totally, 9, 10, 42, 7, 17, and 5 SNPs were suggestive evidence level for $\left(P<1 \times 10^{-5}\right)$ BEHLs at $0.5,1.0,2.0,4.0$, and $8.0 \mathrm{kHz}$ and pure tone average (PTA), respectively. Several promising genetic regions in chromosomes (near the C20orf196, AQPEP, UBQLN3, OR51B5, OR51I2, OR52D1, GLTP, GIT2, and PARK2) nominally associated with ARHI were identified. Gene-based analysis revealed 165, 173, 77, 178, 170, and 145 genes nominally associated with BEHLs at $0.5,1.0,2.0,4.0$, and $8.0 \mathrm{kHz}$ and PTA, respectively $(P<0.05)$. For BEHLs at $0.5,1.0$, and $2.0 \mathrm{kHz}$, the main enriched pathways were phosphatidylinositol signaling system, regulation of ornithine decarboxylase, eukaryotic translation initiation factor (EIF) pathway, amine compound solute carrier (SLC) transporters, synthesis of phosphoinositides (PIPS) at the plasma membrane, and phosphatidylinositols (PI) metabolism. Conclusions. The genetic variations reported herein are significantly involved in functional genes and regulatory domains that mediate ARHI pathogenesis. These findings provide clues for the further unraveling of the molecular physiology of hearing functions and identifying novel diagnostic biomarkers and therapeutic targets of ARHI.
\end{abstract}

\section{Introduction}

Hearing impairment is the most prevalent sensory deficit, affecting over $50 \%$ of middle-aged people and the elderly in China $[1,2]$. Age-related hearing impairment (ARHI) or presbycusis is the most common type of sensorineural hearing loss caused by the natural aging of the auditory system. It is considered an unpreventable and incurable disorder [3].
The typical characteristics of ARHI are gradual progression in later life and bilaterally symmetrical sensorineural hearing impairment, which starts at high frequencies in the early stages and then extends to medium and low frequencies over time. However, early-stage ARHI is often underrecognized. $\mathrm{ARHI}$ is a complex, multifactorial disease that is attributable to confounding genetic and environmental factors $[4,5]$. The genetic predisposition to hearing impairment variations 
approximately have accounted for 25\%-75\% [5-11]. Genome-wide association study (GWAS) has had an enormous impact on our understanding of the molecular physiology of hearing impairment and has allowed the identification of several genetic loci located at or near the GRM7 [12, 13], DCLK1, PTPRD, GRM8, CMIP, ISG20, ACAN, and TRIOBP genes [14].

Only a small part of the genetic variants is explained by known genetic variation and many potential genes to be further discovered. As modern medicine cannot cure ARHI, the active prevention of it is particularly important. The twin study design relies on study twins raising in the same family environment. Simultaneously, on average, dizygotic (DZ) twins share $50 \%$ of the same genes, which can not only be regarded as ordinary sibling pairs but also have perfectly matched ages, prenatal intrauterine environment, and very similar life environment. Therefore, association analysis using DZ twins is more conducive for interpretation of the results. However, the molecular physiology of hearing impairment in middle- and old-aged Chinese population have not been investigated via GWAS yet. This undertaking is important because this population differs from other ethnic populations worldwide in terms of genetic constitutions and lifestyles.

Investigations into genetically related individuals, such as twins, will enhance genetic association studies, and the use of twin-based designs can efficiently identify both common and rare genetic variants underlying complex traits or diseases [15]. A previous study explored the magnitude of genetic impact on better ear hearing levels (BEHLs) and variations in pure tone average (PTA) via twin modelling analyses. Results indicated that heritability estimates range from $47.08 \%$ to $54.20 \%$ for BEHLs within $2.0-12.5 \mathrm{kHz}$ [16]. Owing to the lack of studies on ARHI among middle- and old-aged Chinese twins via GWAS, we further conducted a GWAS to detect the specific genetic variants potentially associated with ARHI. We expect to be able to identify genetic mutations associated with ARHI and elucidate biological processes.

\section{Materials and Methods}

Twin Samples CollectionSamples of twins were collected from the latest genetic epidemiology survey (2012-2013) on previously described aging phenotypes [16-18]. In brief, information was collected via questionnaires and health examination, including anthropometric and laboratory measurements by well-trained clinicians. Participants were excluded if they were unconscious; unable or unwilling to participate; suffering from heart failure, kidney failure, cancer, or severe mental disorders; and currently pregnant or breast feeding; incomplete cotwin pairs were also dropped. Zygosity was determined using 16 multiple short-tandem sequence repeat DNA markers [19-21]. Finally, the samples consisted of 131 complete DZ twins with a median age of 49 years (95\% range: $41-67$ years), of which 128 were females and 134 were males.

This study was approved by the Regional Ethics Committee of the Qingdao CDC Institutional Review Boards. Prior written informed consent was obtained from all participants. The ethical principles of Helsinki Declaration were followed.

2.1. Audiometric Examination. Audiometric examination was performed following the method described in a previous study [16]. In brief, the twins underwent otoscopy, and then, the pure-tone air-conducted hearing thresholds in each ear were separately measured at $0.5,1.0,2.0,4.0$, and $8.0 \mathrm{kHz}$ by using a diagnostic audiometer. BEHL was then calculated as the lower value of both ears at each frequency. Finally, the PTA at $0.5,1.0,2.0,4.0$, and $8.0 \mathrm{kHz}$ was separately calculated for the left and the right ear, and the better ear (i.e., the one with the lower value) was selected.

2.2. Genotyping and Quality Control. Genomic DNA was first extracted from the whole peripheral blood of the $131 \mathrm{DZ}$ twins by using QIAamp DNA Blood Mini Kit (Qiagen $\mathrm{GmbH}$, Hilden, Germany). Quantity and integrity of genomic DNA were then determined. Subsequently, DNA samples were genotyped on the Illumina's Infinium Omni2.5Exome-8v1.2 Bead Chip platform (Illumina, San Diego, CA, USA). Autosome and chromosome X data were analyzed. Quality control was applied using the following criteria: call rate $>0.98$, minor allele frequency $>0.01$, Hardy - Weinberg Equilibrium $>1 \times$ $10^{-4}$, and locus missing $<0.05$ according to genome-wide efficient mixed-model association (GEMMA) [22]. Linear-Mixed Models were used to test the genotype-phenotype association by using GEMMA. Genetic relationship matrix was included in the model analyses because of our twin pedigree data. Finally, a total of 1,365,315 single-nucleotide polymorphisms (SNPs) qualified for subsequent analyses.

\subsection{Statistical Analysis}

2.3.1. Basic Characteristics Analysis. Descriptive statistics were computed using SPSS version 22.0. Square-root transformation for BEHLs and rank transformation for PTA were performed for normality. We first performed a normality test for basic characteristics. For those that did not conform to the normal distribution, the Mann-Whitney test was used for comparison.

2.3.2. SNP-Based Analysis. The association between ARHI and SNP genotypes across the genome was tested using the GEMMA software [22]. Sex, age, educational level, and the first five principal components served as covariates in model fitting. SNPs that reached a suggestive evidence level $\left(P<1 \times 10^{-5}\right)$ rather than the conventional genome-wide significance level $\left(P<5 \times 10^{-8}\right)$ for the association were detected $[23,24]$. The chromosome $\mathrm{X}$-wide association study (XWAS) was used to find the possible trait association signals from chromosome X. Functional elaboration of the detected SNPs was further performed, and likely, cell types of action were predicted using the HaploReg v4.1 software [25, 26]. Enrichment results of cell type enhancers were reported (uncorrected $P<0.05$ ).

2.3.3. Gene-Based Analysis. Gene-based analysis was implemented using SNP-set association test via the versatile gene-based association study-2 (VEGAS2) approach, which 
TABle 1: Descriptive statistics for dizygotic twin pairs by gender.

\begin{tabular}{lccr}
\hline Traits $^{\#}$ & Male $(n=134)$ & Female $(n=128)$ & All $(n=262)$ \\
\hline Age, years & $50.00(41.00-67.80)$ & $49.00(41.00-68.40)$ & $49.00(41.00-67.28)$ \\
$B E H L, d B$ & & & \\
$0.5 \mathrm{kHz}$ & $20.00(5.00-43.00)$ & $20.00(5.00-40.00)$ & $20.00(5.00-40.00)$ \\
$1.0 \mathrm{kHz}$ & $15.00(0.00-38.00)$ & $15.00(0.00-34.00)$ & $15.00(0.00-35.00)$ \\
$2.0 \mathrm{kHz}$ & $10.00(0.00-51.00)$ & $10.00(0.00-34.00)$ & $10.00(0.00-35.00)$ \\
$4.0 \mathrm{kHz}$ & $25.00(2.00-78.00)$ & $15.00(0.00-50.00)^{*}$ & $15.00(0.00-70.00)$ \\
$8.0 \mathrm{kHz}$ & $25.00(5.00-83.00)$ & $15.00(0.00-60.00)^{*}$ & $20.00(0.00-77.13)$ \\
PTA & $20.00(7.00-46.00)$ & $15.00(3.20-38.60)^{*}$ & $17.00(5.00-43.85)$ \\
\hline
\end{tabular}

BEHL: better ear hearing level; PTA: pure tone average ${ }^{*} P<0.001^{*}$ traits were described as median (2.5\%-97.5\% quantiles).

incorporated information from a full set of GWAS summary data within one gene and accounts for linkage disequilibrium between them $[27,28]$. SNPs from "1000G East Asian Population" were adopted. $P<0.05$ was considered as nominal significance level [29].

2.3.4. Pathway Enrichment Analysis. Pathway enrichment analysis was conducted using pathway scoring algorithm (PASCAL) [30, 31]. First, the location of genetic marker SNPs in the genes was determined, and the related scores of all genes in the pathway were calculated. Chi-squared or empirical scores were used to evaluate the pathway enrichment of high-scoring (possibly fused) genes, avoiding any standard binary enrichment test with inherent $P$ value threshold. The pathway and its corresponding genes were selected KEGG, Reactome, and Biocarta.

\section{Results}

3.1. Basic Characteristics. The basic characteristics of the 131 DZ twins were summarized in Table 1 . The males showed a higher moderate and high BEHLs $(4.0$ and $8.0 \mathrm{kHz})$ and PTA than the females $(P<0.001)$, whereas no difference was found in terms of low BEHLs $(0.5$ and $1.0 \mathrm{kHz})$.

3.2. SNP-Based Analysis. A total of $1,365,315$ SNPs genotyped from the current sample were included in the GWAS. The relationships between the observed and expected GWAS $P$ values for BEHLs and PTA were illustrated in quantilequantile ( $Q-Q)$ plots (Figure 1). The values of $\lambda$-statistic were close to one (0.9906-1.0110), suggesting no evidence of bias from population stratification or genomic inflation of the test statistics. The slight deviation in the upper right tail from null distribution crudely suggested some form of associations.

As illustrated in Manhattan plots (Figure 2), rs6633657 was the only SNP that reached the genome-wide significance level $\left(P=1.19 \times 10^{-8}\right)$. This SNP was located in the intron region of PTCHD1-AS on chromosome 23 for BEHL at $2.0 \mathrm{kHz}$. Particular for the trait association signals from chromosome $\mathrm{X}$, then we ascertained by using the XWAS. By analyzing the associations of rs6633657, we identified this SNP was associated with $\mathrm{BEHL}_{2.0}$ (Additional file 1). No other SNP reached the genome-wide significance level $\left(P<5 \times 10^{-8}\right)$ for BEHLs at the other frequencies and PTA.
However, 9, 10, 42, 7, and 17 SNPs were suggestive of association $\left(P<1 \times 10^{-5}\right)$ for BEHLs at $0.5,1.0,2.0,4.0$, and $8.0 \mathrm{kHz}$, respectively; by comparison, five SNPs were suggestive of association for PTA (Table 2). No consistent SNPs were observed for BEHLs at $0.5,1.0,2.0,4.0$, and $8.0 \mathrm{kHz}$ frequencies.

As illustrated by the regional association plots (Figure 3), several chromosomal loci showed nominal association with ARHI. Among these top signals (Table 2), three SNPs $\left(P=6.25 \times 10^{-7}-2.15 \times 10^{-6}\right)$ were located at or near the C20orf196 gene on chromosome 20p12.3 for BEHL at $0.5 \mathrm{kHz}$ (Figure 3(a)); four SNPs $\left(P=2.93 \times 10^{-7}-7.90 \times\right.$ $\left.10^{-6}\right)$ at or near the $A Q P E P$ gene on chromosome $5 \mathrm{q} 23.1$ for BEHL at $1.0 \mathrm{kHz}$ (Figure 3(b)); nine SNPs $(P=9.81 \times$ $\left.10^{-7}-8.33 \times 10^{-6}\right)$ at or near the UBQLN3, OR51B5, OR51I2, and OR52D1 genes on chromosome 11p15.4 for BEHL at $2.0 \mathrm{kHz}$ (Figure 3(c)); two SNPs $\left(P=1.05 \times 10^{-6}\right.$ and $\left.9.08 \times 10^{-6}\right)$ at or near the GLTP and GIT2 genes on chromosome 12q24.11 for BEHL at $4.0 \mathrm{kHz}$ (Figure 3(d)); and six SNPs $\left(P=5.44 \times 10^{-7}-7.38 \times 10^{-6}\right)$ at or near the PARK2 gene on chromosome $6 \mathrm{q} 26$ for BEHL at $8.0 \mathrm{kHz}$ (Figure $3(\mathrm{e}))$. For PTA, two SNPs $\left(P=2.34 \times 10^{-6}\right.$ and 5.02 $\times 10^{-6}$ ) were positioned within or closest to the GLTP and GIT2 genes on chromosome 12q24.11 (Figure 3(f)).

The primary $\mathrm{T}$ helper memory/regulatory cells from peripheral blood was identified for BEHL at $0.5 \mathrm{kHz}$ by using the HaploReg v4.1 software (Additional file 2). The results were compared with meaningful ARHI-associated SNPs previously reported by other GWAS. No evidence of replication was found.

3.3. Gene-Based Analysis. A total of $165,173,77,178,170$, and 145 genes were observed to be nominally associated with BEHLs at $0.5 \mathrm{kHz}, 1.0 \mathrm{kHz}, 2.0 \mathrm{kHz}, 4.0 \mathrm{kHz}$, and $8.0 \mathrm{kHz}$ and PTA, respectively $(P<0.05)$ (Additional file 3$)$. The top 20 genes for $\mathrm{BEHL}_{0.5}$ ranked by $P$ values are listed in Table 3 $\left(\mathrm{BEHL}_{1.0}\right.$ : Additional file 4, $\mathrm{BEHL}_{2.0}$ : Additional file 5, $\mathrm{BEHL}_{4.0}$ : Additional file 6, $\mathrm{BEHL}_{8.0}$ : Additional file 7, PTA: Additional file 8). C20orf196 gene for $0.5 \mathrm{kHz}, S L C 16 A 9$, UBQLN3, and OR51I2A genes for $2.0 \mathrm{kHz}$ and FAM184A and TBC1D1genes for $8.0 \mathrm{kHz}$ had already been shown in suggestive level SNP-based. 


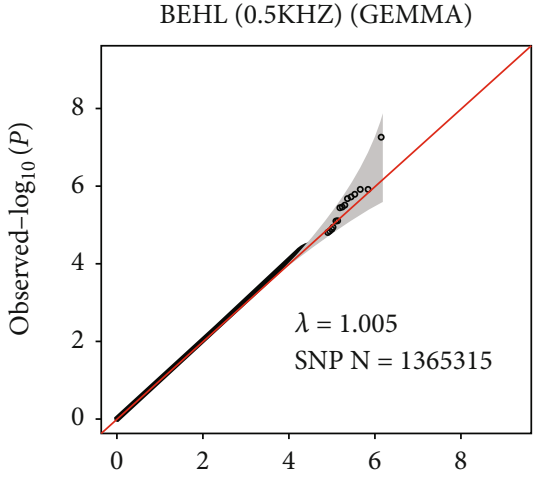

Expected- $\log _{10}(P)$

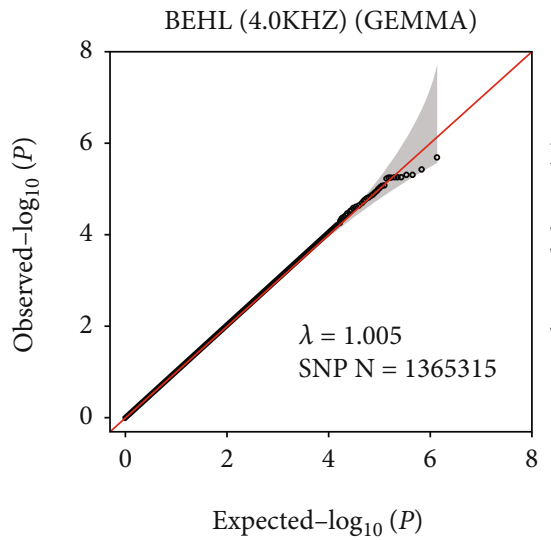

BEHL (1.0KHZ) (GEMMA)

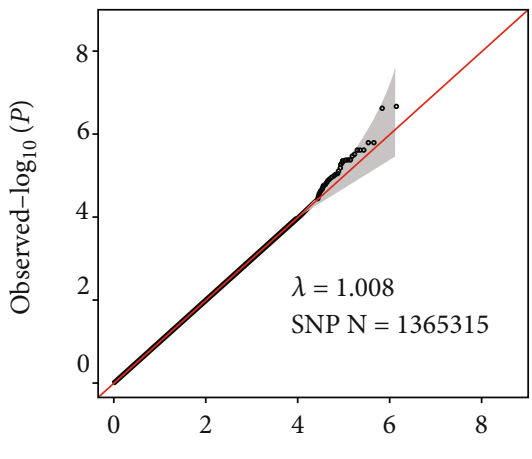

Expected- $\log _{10}(P)$

BEHL (8.0KHZ) (GEMMA)

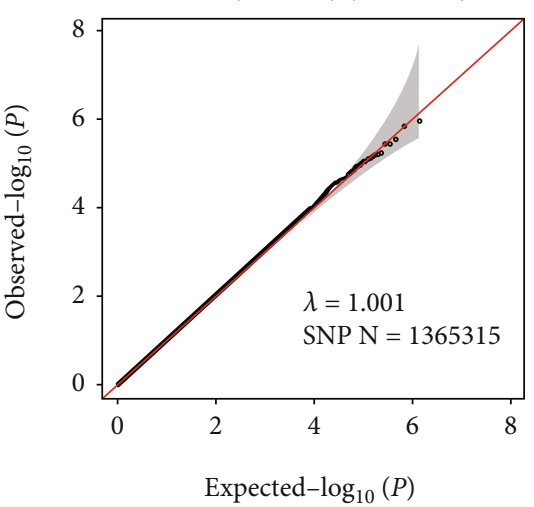

BEHL (2.0KHZ) (GEMMA)

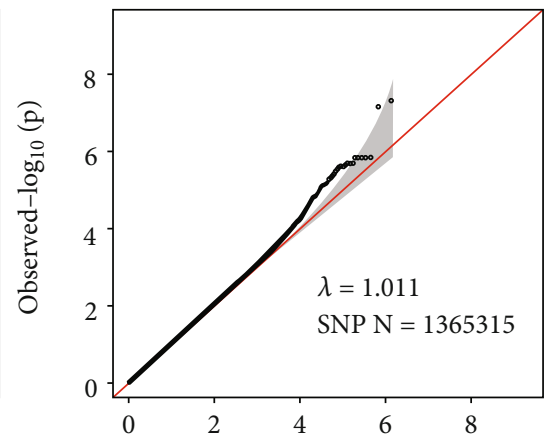

Expected- $\log _{10}(P)$

PTAs (means) (GEMMA)

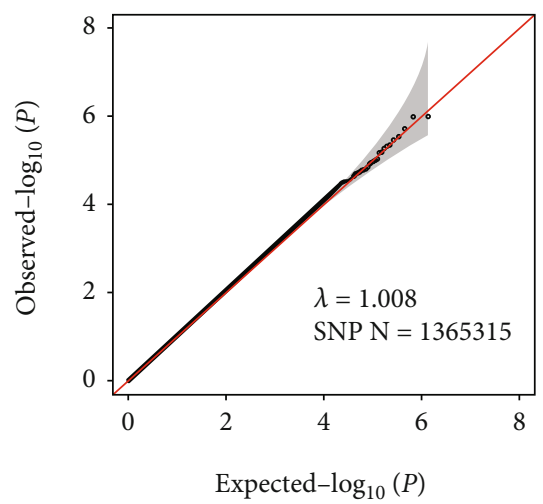

FIGURE 1: Quantile-quantile plots for GWAS of age-related hearing impairment measured by better ear hearing levels and pure tone average. The $x$-axis shows the - $\log 10$ of expected $P$ values of association from Chi-square distribution, and the $y$-axis shows the -log 10 of $P$ values from the observed Chi-square distribution. Black dots represent the observed data with the top hit single-nucleotide polymorphism (SNP) being colored, and the red line is the expectation under the null hypothesis of no association. Gene at the best SNP is indicated.
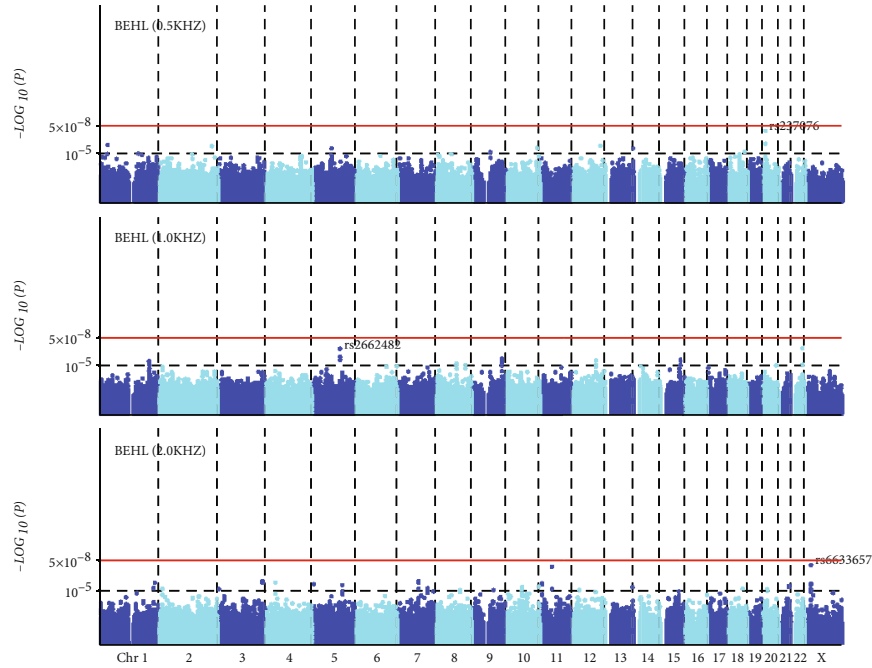
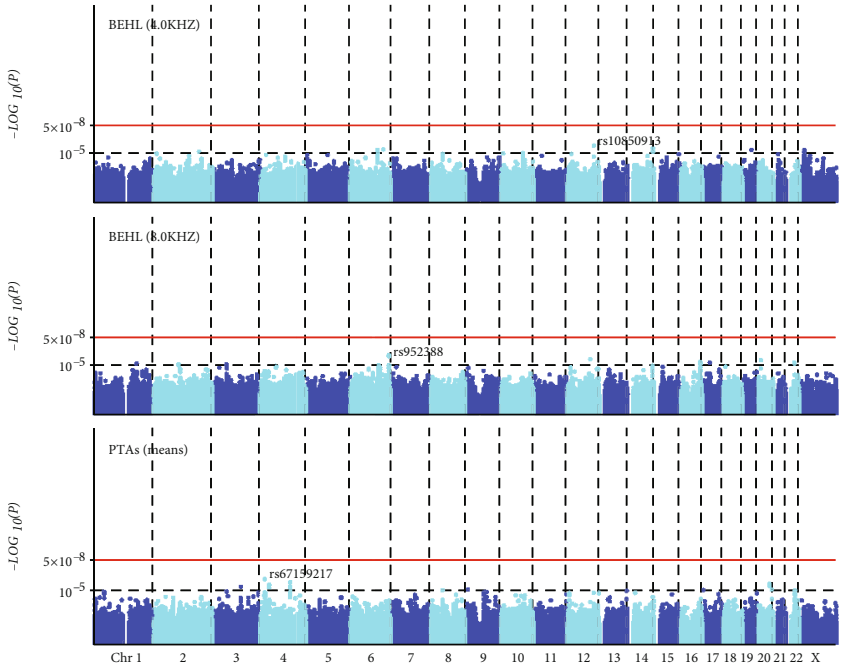

FIgURE 2: Manhattan plots for GWAS of age-related hearing impairment measured by better ear hearing levels (BEHLs) and pure tone average (PTA). The $x$-axis shows the numbers of autosomes and the $\mathrm{X}$ chromosome, and the $y$-axis shows the -log10 of $P$ values for statistical significance. The dots represent the single-nucleotide polymorphisms (SNPs). Except for the strongest association being detected with rs6633657 $\left(P=1.19 \times 10^{-8}\right)$ located on chromosome 23 for BEHL $(2.0 \mathrm{kHz})$, no other SNP reached the genome-wide significance level $\left(P<5 \times 10^{-8}\right)$. However, several SNPs were suggestive of association $\left(P<1 \times 10^{-5}\right)$ for BEHLs and PTA. 
TABLE 2: Summary of SNPs associated with ARHI measured by BEHLs and PTA in GWAS $\left(P\right.$ value $\left.<1 \times 10^{-5}\right)$.

\begin{tabular}{|c|c|c|c|c|c|}
\hline Traits & SNP & $\mathrm{CHR}$ & $\mathrm{BP}$ & $P$ value & Closest genes or genes \\
\hline \multicolumn{6}{|c|}{$\mathrm{BEHL}$, by frequency $(\mathrm{kHz})$} \\
\hline \multirow{9}{*}{0.5} & rs237076 & 20 & 5810831 & $6.25 E-07$ & C20orf196 \\
\hline & rs6790988 & 3 & 170263320 & $1.80 E-06$ & CLDN11 \\
\hline & rs55705402* & 20 & 5828630 & $2.15 E-06$ & C20orf196 \\
\hline & rs55827353* & 20 & 5826245 & $2.15 E-06$ & C20orf196 \\
\hline & kgp8299849 (rs77570135) & 10 & 123472528 & $2.84 E-06$ & LOC440700 \\
\hline & rs 10753110 & 1 & 175869554 & $4.68 E-06$ & LOC107985228 \\
\hline & rs9293725 & 5 & 77177468 & $5.48 E-06$ & LOC101929154 \\
\hline & rs1908968 & 4 & 143268702 & $7.89 E-06$ & $I N P P 4 B$ \\
\hline & rs12194558 & 6 & 153106506 & $9.11 E-06$ & LOC105378065 \\
\hline \multirow{10}{*}{1.0} & rs2662482 ${ }^{\#}$ & 5 & 115358663 & $2.93 E-07$ & $L V R N$ (also known as $A Q P E P$ ) \\
\hline & kgp928895 (rs201156432) & 22 & 43742432 & $3.35 E-07$ & SCUBE1 \\
\hline & rs $2560690^{*}$ & 5 & 115354702 & $4.07 E-06$ & $L V R N$ (also known as $A Q P E P$ ) \\
\hline & rs2662464* & 5 & 115356017 & $4.07 E-06$ & $L V R N$ (also known as $A Q P E P$ ) \\
\hline & rs73166130 & 22 & 43746132 & $4.17 E-06$ & SCUBE1 \\
\hline & rs35577903 & 1 & 209277772 & $5.68 E-06$ & LOC105372895; LOC107985255 \\
\hline & rs9460076 & 6 & 170517603 & $7.49 E-06$ & RPL12P23 \\
\hline & rs11106865 & 12 & 93515552 & $7.73 E-06$ & LOC643339 \\
\hline & rs $2560687 *$ & 5 & 115360342 & $7.90 E-06$ & $L V R N$ (also known as $A Q P E P$ ) \\
\hline & rs11108973 & 12 & 97721824 & $1.01 E-05$ & LINC02409 \\
\hline \multirow{26}{*}{2.0} & rs6633657 & 23 & 22836669 & $1.19 E-08$ & PTCHD1-AS \\
\hline & rs1974517 & 23 & 22837661 & $6.64 E-07$ & PTCHD1-AS \\
\hline & rs2234456 & 11 & 5529139 & $9.81 E-07$ & $U B Q L N 3$ \\
\hline & rs $1498482^{*}$ & 11 & 5483235 & $1.08 E-06$ & OR51B5 \\
\hline & rs10838135* & 11 & 5473913 & $1.11 E-06$ & OR51B5; OR51I2 \\
\hline & rs $12420260^{*}$ & 11 & 5473418 & $1.11 E-06$ & OR51B5; OR51I2 \\
\hline & rs $1603776 *$ & 11 & 5479891 & $1.11 E-06$ & OR51B5 \\
\hline & rs7801592 & 7 & 85187817 & $1.13 E-06$ & LINC00972 \\
\hline & rs66808307 & 7 & 85199500 & $1.13 E-06$ & LINC00972 \\
\hline & rs73189269 & 7 & 85199160 & $1.13 E-06$ & LINC00972 \\
\hline & rs112837279 & 7 & 85203112 & $1.13 E-06$ & LINC00972 \\
\hline & rs11037503* & 11 & 5475597 & $1.23 E-06$ & OR51B5; OR51I2 \\
\hline & rs5029981 & 3 & 186438314 & $1.47 E-06$ & KNG1 \\
\hline & rs6950989 & 7 & 85197091 & $1.52 E-06$ & LINC00972 \\
\hline & kgp22739604 (rs6629497) & 23 & 22846422 & $1.53 E-06$ & PTCHD1-AS \\
\hline & rs630428 & 4 & 38121363 & $1.64 E-06$ & TBC1D1 \\
\hline & rs 17584191 & 5 & 125533130 & $1.79 E-06$ & LINC02039 \\
\hline & rs 3763747 & 10 & 61412335 & $1.94 E-06$ & SLC16A9 \\
\hline & kgp22746837 (rs12688139) & 23 & 22834788 & $2.04 E-06$ & PTCHD1-AS \\
\hline & rs2242206 & 10 & 61414011 & $3.36 E-06$ & SLC16A9 \\
\hline & rs1007490 & 23 & 22835253 & $3.73 E-06$ & PTCHD1-AS \\
\hline & rs 12412363 & 10 & 130571475 & $4.23 E-06$ & LOC105378555 \\
\hline & rs1972703 & 3 & 186463343 & $5.49 E-06$ & KNG1 \\
\hline & kgp9174891 (rs201551669) & 5 & 125549376 & $6.32 E-06$ & LINC02039 \\
\hline & rs3827672 & 9 & 115924811 & $6.50 E-06$ & SLC31A2 \\
\hline & rs 12106130 & 20 & 15616247 & $6.65 E-06$ & MACROD2 \\
\hline
\end{tabular}


TABLE 2: Continued.

\begin{tabular}{|c|c|c|c|c|c|}
\hline Traits & SNP & $\mathrm{CHR}$ & $\mathrm{BP}$ & $P$ value & Closest genes or genes \\
\hline & rs4948351 & 10 & 61425189 & $6.70 E-06$ & SLC16A9 \\
\hline & kgp22769801 (rs6629500) & 23 & 22847169 & $6.77 E-06$ & PTCHD1-AS \\
\hline & rs17160047 & 7 & 85203213 & $6.88 E-06$ & LINC00972 \\
\hline & rs7729369 & 5 & 125544313 & $6.92 E-06$ & LINC02039 \\
\hline & rs7101919* & 11 & 5510688 & $7.30 E-06$ & OR51B5; OR52D1 \\
\hline & rs 10826342 & 10 & 61433292 & $7.40 E-06$ & SLC16A9 \\
\hline & rs1171606 & 10 & 61434519 & $7.40 E-06$ & SLC16A9 \\
\hline & kgp1872947 (rs199963695) & 8 & 96237951 & $7.64 E-06$ & LINC01298 \\
\hline & rs7728158 & 5 & 125602203 & $7.88 E-06$ & LOC101927488 \\
\hline & rs6135472 & 20 & 15618014 & $7.98 E-06$ & MACROD2 \\
\hline & rs $4638331^{*}$ & 11 & 5511431 & $8.21 E-06$ & OR51B5; OR52D1 \\
\hline & rs72881227* & 11 & 5498649 & $8.33 E-06$ & OR51B5 \\
\hline & rs 4509290 & 8 & 96237596 & $8.49 E-06$ & LINC01298 \\
\hline & rs56394481 & 13 & 110019360 & $8.53 E-06$ & LOC105370359 \\
\hline & rs4293213 & 12 & 23923620 & $9.62 E-06$ & SOX5 \\
\hline & rs11592061 & 10 & 3132945 & $9.70 E-06$ & PFKP \\
\hline \multirow{6}{*}{4.0} & rs10850913 & 12 & 110312232 & $1.05 E-06$ & GLTP \\
\hline & rs35515683 & 6 & 113848824 & $5.20 E-06$ & LINC02541 \\
\hline & rs 10872099 & 6 & 113926484 & $5.39 E-06$ & LINC02541 \\
\hline & rs80050647 & 10 & 7877888 & $5.40 E-06$ & TAF3 \\
\hline & rs7145420 & 14 & 101915881 & $7.77 E-06$ & LINC02314 \\
\hline & kgp3400527 (rs79297719) & 10 & 91681180 & $8.39 E-06$ & LINC01375; LOC105378425 \\
\hline \multirow{18}{*}{8.0} & rs2292354* & 12 & 110368201 & $9.08 E-06$ & GIT2 \\
\hline & rs2096982 & 6 & 162660989 & $5.44 E-07$ & PARK2 \\
\hline & rs952388* & 6 & 162716541 & $7.04 E-07$ & PARK2 \\
\hline & rs2309938 & 2 & 101699594 & $1.01 E-06$ & TBC1D8 \\
\hline & rs13388167 & 2 & 101724667 & $1.89 E-06$ & TBC1D8 \\
\hline & rs718772 & 22 & 30504207 & $2.61 E-06$ & HORMAD2 \\
\hline & rs9625919 & 22 & 30500958 & $2.84 E-06$ & HORMAD2 \\
\hline & rs11080090 & 17 & 27502029 & $3.70 E-06$ & MYO18A \\
\hline & rs10945825* & 6 & 162809009 & $4.27 E-06$ & PARK2 \\
\hline & rs10945826* & 6 & 162811902 & $4.31 E-06$ & PARK2 \\
\hline & rs $10455904^{*}$ & 6 & 162820082 & $4.31 E-06$ & PARK2 \\
\hline & rs2247304 & 12 & 96424665 & $4.36 E-06$ & $L T A 4 H$ \\
\hline & rs12598984 & 16 & 82521687 & $6.10 E-06$ & LOC101928392 \\
\hline & rs12143791 & 1 & 178071170 & $6.55 E-06$ & RASAL2 \\
\hline & rs $10455908^{*}$ & 6 & 162843312 & $7.38 E-06$ & $P A R K 2$ \\
\hline & rs7745460 & 6 & 119396199 & $7.79 E-06$ & FAM184A \\
\hline & rs 10871427 & 16 & 82521530 & $8.35 E-06$ & LOC101928392 \\
\hline & rs2357161 & 6 & 119412952 & $8.63 E-06$ & FAM184A \\
\hline \multirow{5}{*}{ PTA } & rs10850913 & 12 & 110312232 & $2.34 E-06$ & $G L T P$ \\
\hline & rs4565962 & 12 & 110264903 & $2.57 E-06$ & TRPV4 \\
\hline & rs2292354* & 12 & 110368201 & $5.02 E-06$ & GIT2 \\
\hline & rs11931969 & 4 & 22235368 & $5.07 E-06$ & LOC100505912 \\
\hline & rs2309938 & 2 & 101699594 & $9.56 E-06$ & TBC1D8 \\
\hline
\end{tabular}

kgp: 1000 genomes project; ${ }^{*}$ : represented the top signals illustrated in the regional association plots for BEHLs at each frequency and for PTA; ${ }^{*}$ represented the SNPs showing linkage disequilibrium (LD) with the top signals for BEHLs at each frequency and for PTA. 


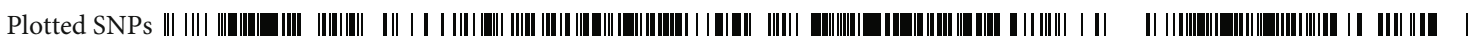
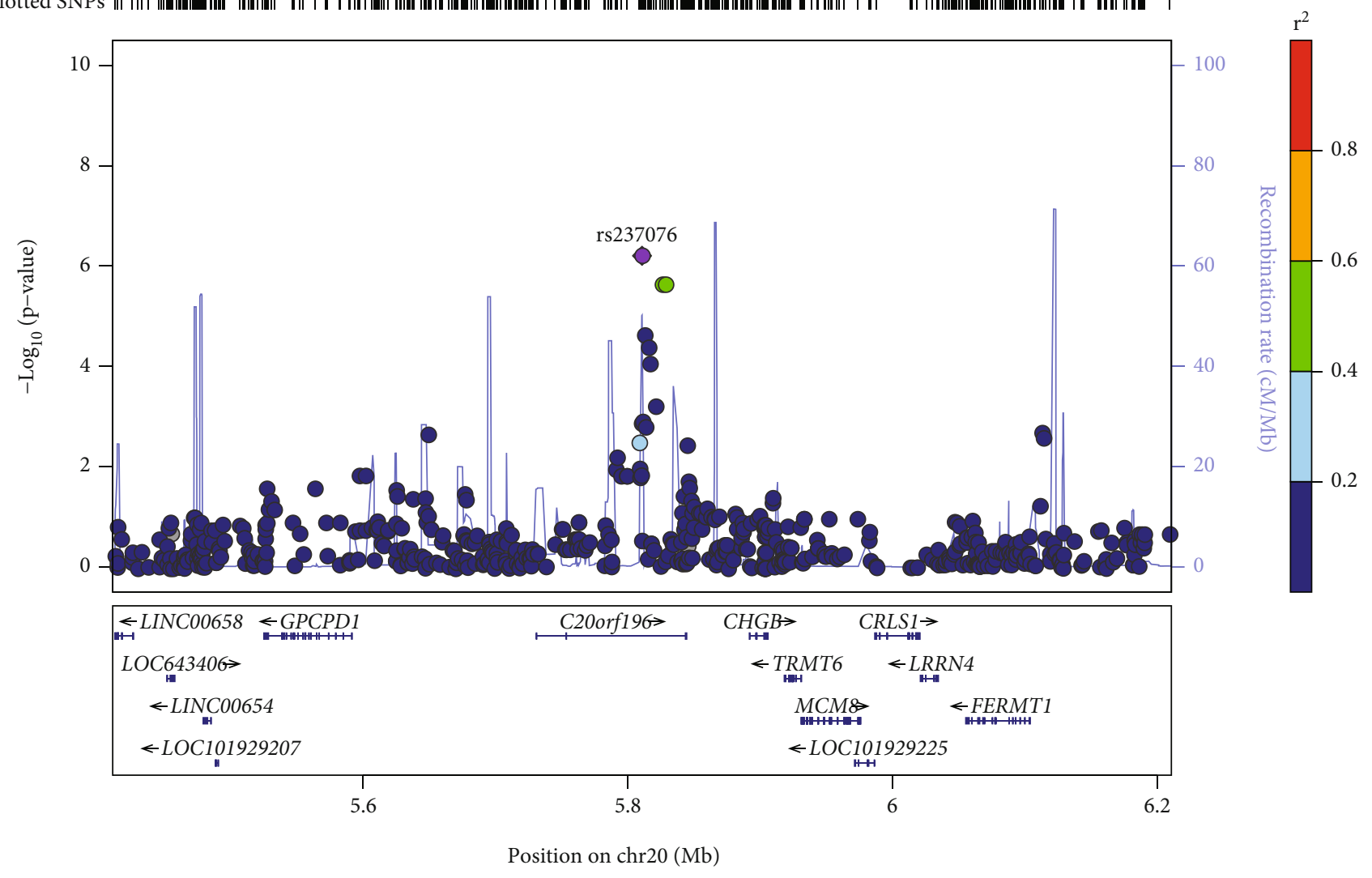

(a)

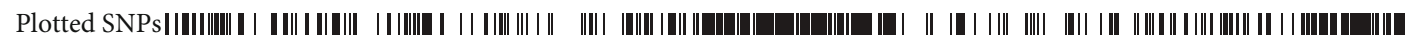

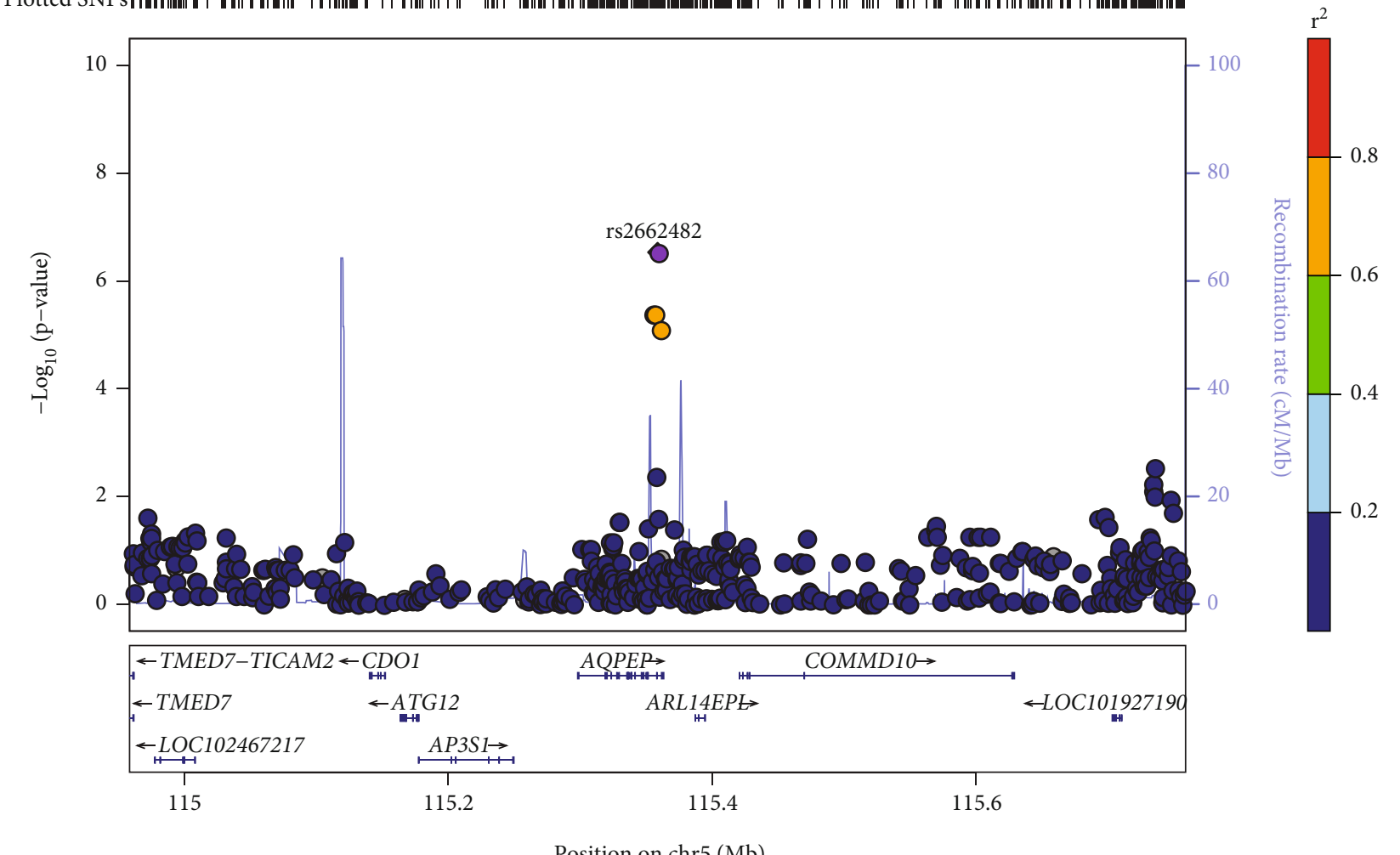

Position on $\operatorname{chr} 5(\mathrm{Mb})$

(b)

Figure 3: Continued. 


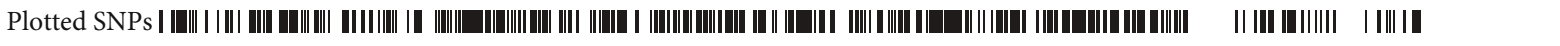

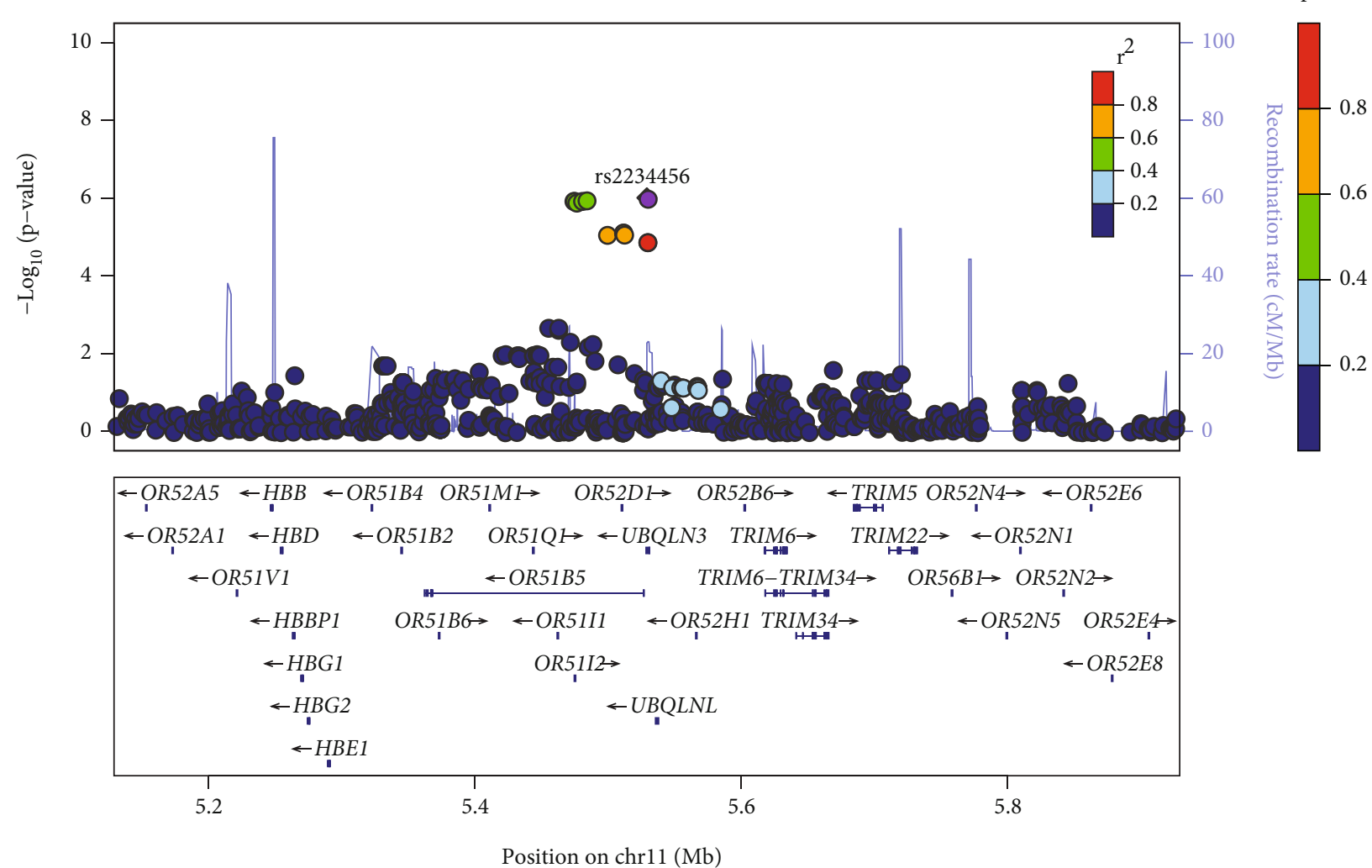

(c)

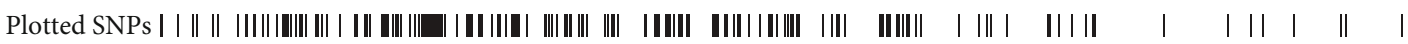

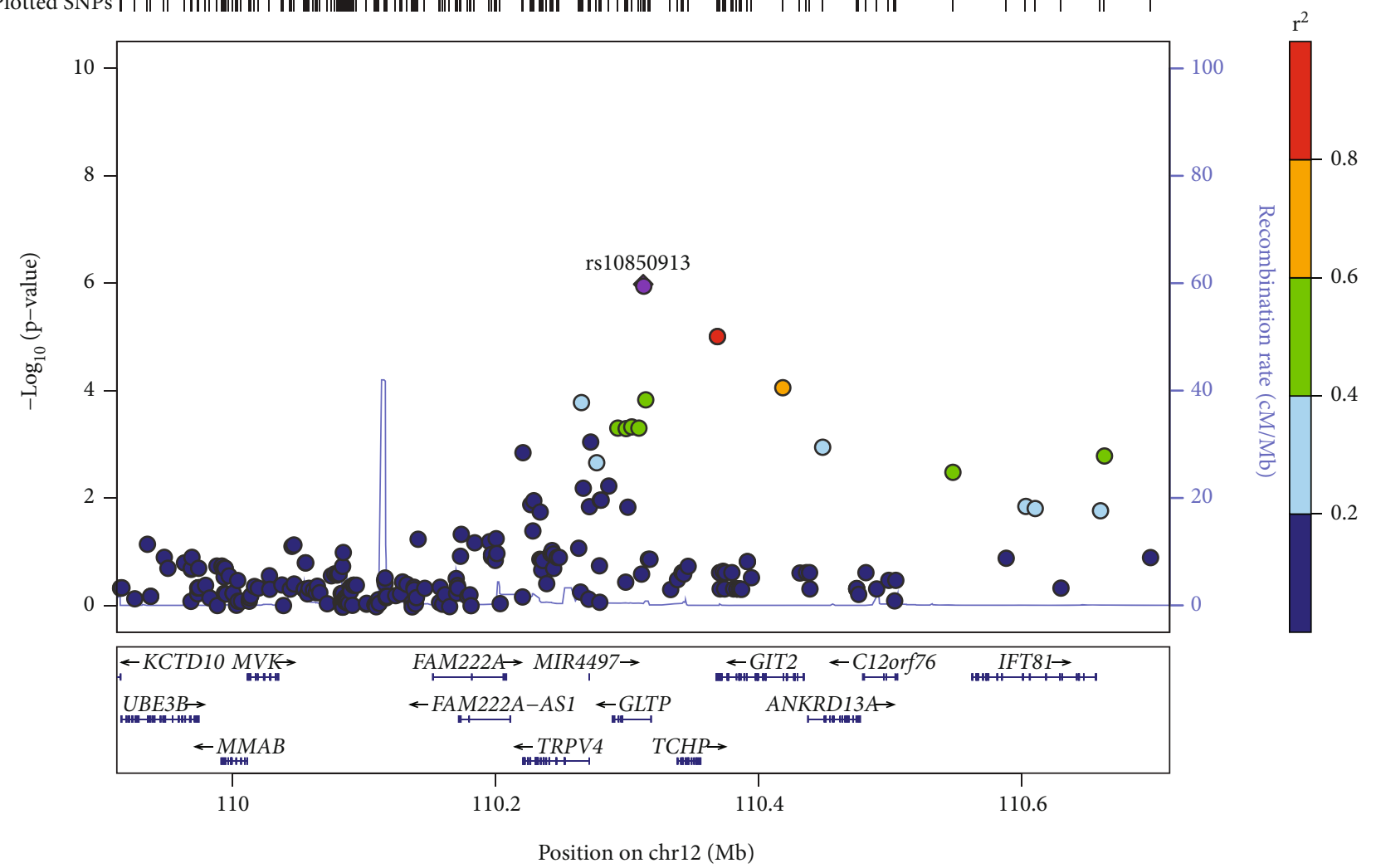

(d)

FIgURe 3: Continued. 


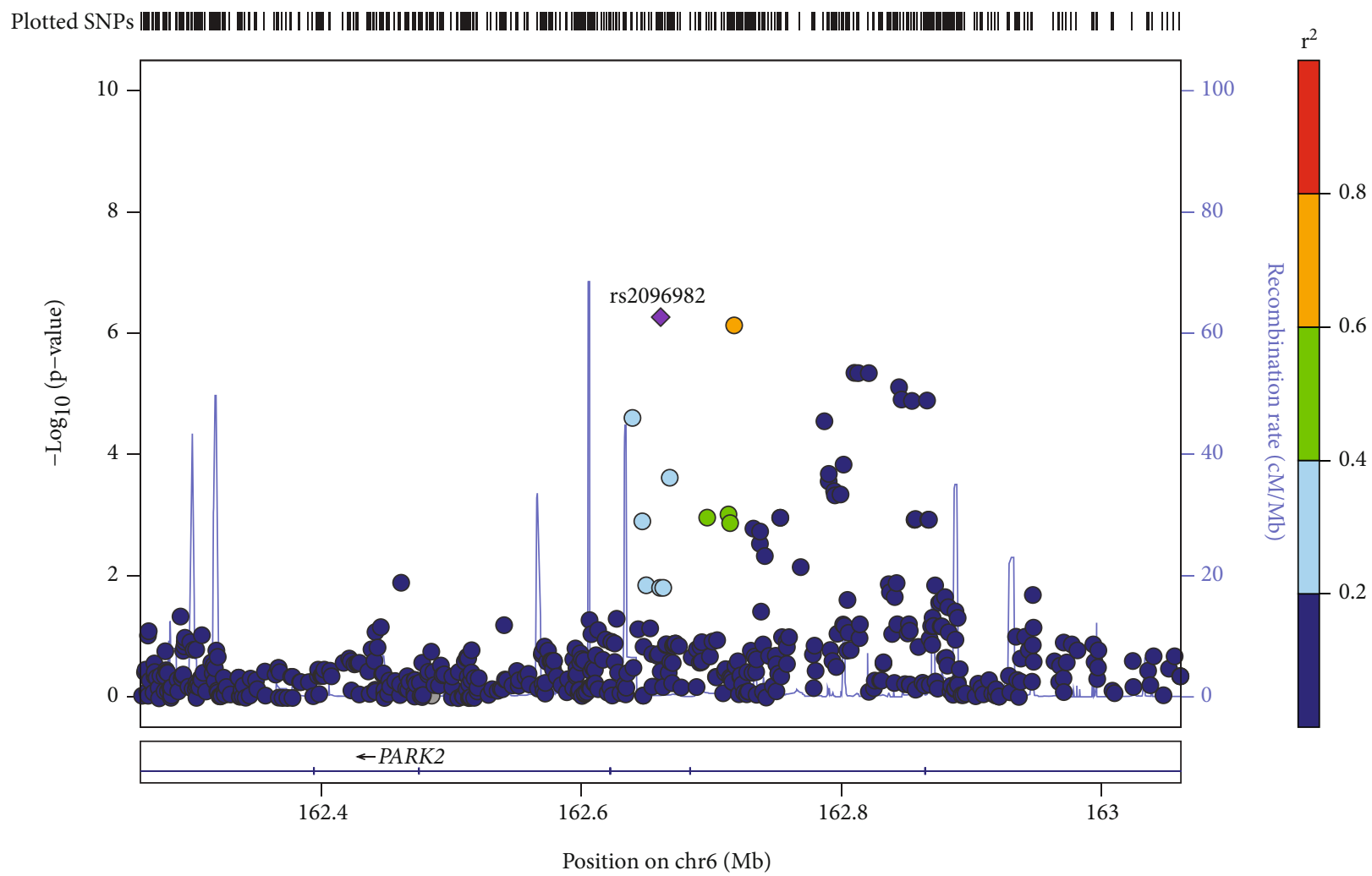

(e)

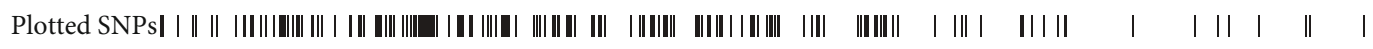
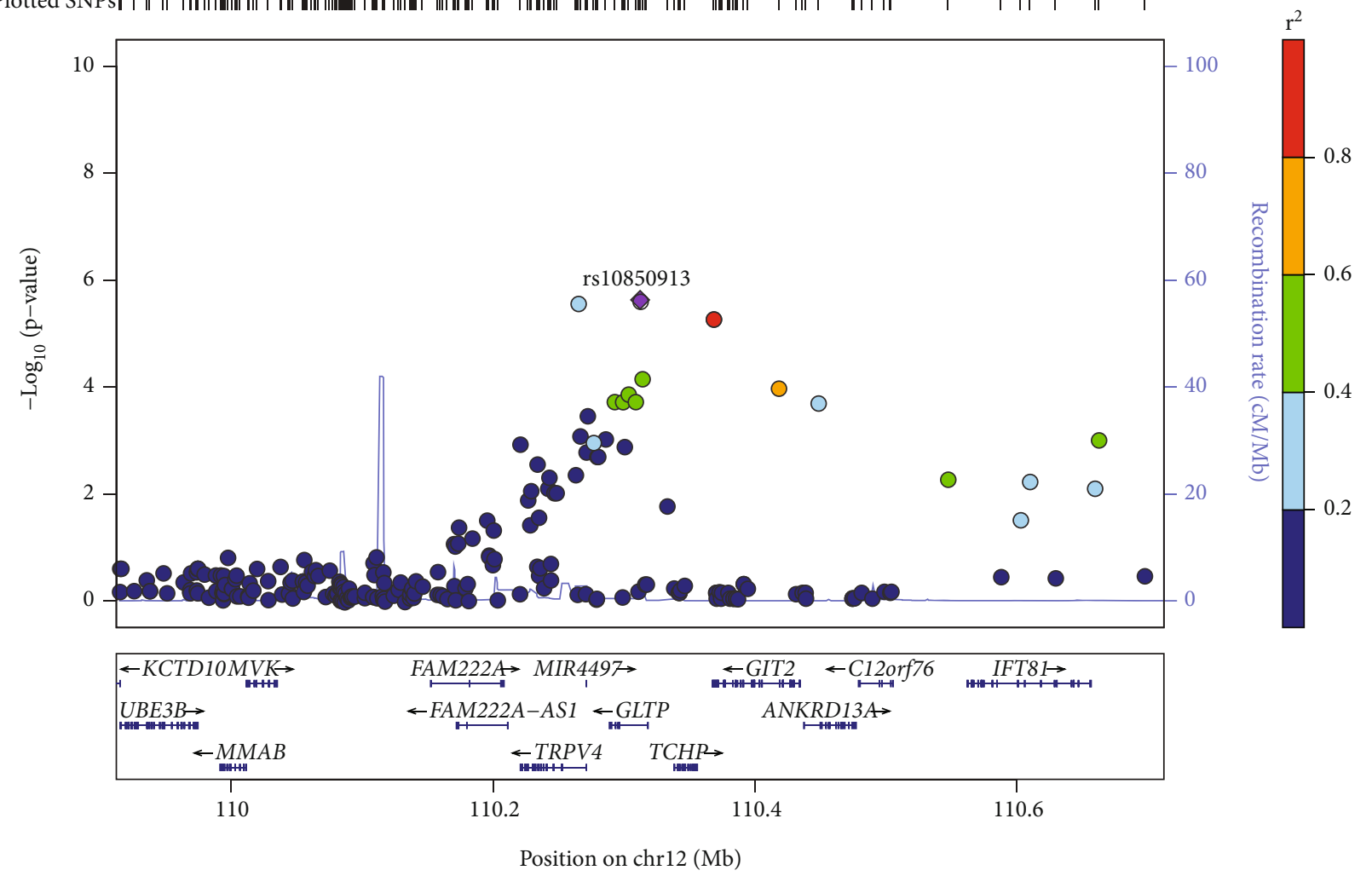

(f)

FIgURE 3: Regional association plots showing strong signals for GWAS of age-related hearing impairment measured by better ear hearing levels (BEHLs) and pure tone average (PTA). (a) $\mathrm{BEHL}_{(0.5 \mathrm{kHz})}$, (b) $\mathrm{BEHL}_{(1.0 \mathrm{kHz})}$, (c) $\mathrm{BEHL}_{(2.0 \mathrm{kHz})}$, (d) $\mathrm{BEHL}_{(4.0 \mathrm{kHz})}$, (e) $\mathrm{BEHL}_{(8.0 \mathrm{kHz})}$, and (f) PTA. 
TABLE 3: Top 20 genes from VEGAS2 gene-based analysis showing the strongest association with $\mathrm{BEHL}_{0.5}$.

\begin{tabular}{|c|c|c|c|c|c|c|c|c|}
\hline Chr & Gene & nSNPs & Start position & Stop position & Gene-based test statistic & $P$ value & Top-SNP & Top-SNP $P$ value \\
\hline 17 & RNASEK-C17orf49 & 6 & 6915735 & 6920843 & 70.85 & $2.00 E-05$ & rs7338 & $8.40 E-05$ \\
\hline 17 & C17orf49 & 5 & 6918055 & 6920843 & 55.38 & $2.20 E-05$ & rs14309 & $1.70 E-04$ \\
\hline 20 & C20orf196 & 72 & 5731042 & 5844559 & 273.82 & $6.00 E-05$ & rs237076 & $6.20 E-07$ \\
\hline 17 & MIR497HG & 3 & 6919136 & 6922973 & 30.88 & $1.50 E-04$ & rs11078662 & $2.20 E-04$ \\
\hline 19 & MYO1F & 26 & 8585673 & 8642331 & 115.33 & $2.80 E-04$ & rs3213834 & $2.60 E-05$ \\
\hline 16 & ITGAM & 11 & 31271287 & 31344213 & 135.61 & $3.70 E-04$ & rs4594268 & $9.00 E-05$ \\
\hline 3 & SLC7A14 & 64 & 170177341 & 170303863 & 263.01 & $5.10 E-04$ & rs6790988 & $1.80 E-06$ \\
\hline 12 & CAPS2 & 28 & 75669758 & 75784702 & 157.74 & $5.20 E-04$ & rs12367329 & $8.10 E-05$ \\
\hline 7 & SAMD9 & 14 & 92728825 & 92747336 & 75.2 & $5.60 E-04$ & rs76427362 & $4.80 E-03$ \\
\hline 4 & $M G A R P$ & 7 & 140187316 & 140201492 & 37.55 & $5.80 E-04$ & rs13120574 & $1.20 E-03$ \\
\hline 12 & GLIPR1L1 & 8 & 75728462 & 75764169 & 48.74 & $6.10 E-04$ & rs12367329 & $8.10 E-05$ \\
\hline 22 & $M L C 1$ & 24 & 50497819 & 50524358 & 132.45 & $6.10 E-04$ & rs5771144 & $1.10 E-04$ \\
\hline 18 & $R B F A$ & 7 & 77794345 & 77810652 & 40.58 & $6.20 E-04$ & rs3744873 & $1.60 E-03$ \\
\hline 23 & GPR50 & 2 & 150345055 & 150349937 & 14.76 & $6.50 E-04$ & rs2072621 & $3.30 E-03$ \\
\hline 14 & GPR132 & 8 & 105515725 & 105531887 & 34.35 & $6.70 E-04$ & rs7147439 & $3.40 E-03$ \\
\hline 15 & STARD9 & 66 & 42867856 & 43013196 & 253.11 & $6.80 E-04$ & rs61192504 & $6.40 E-04$ \\
\hline 11 & $L G R 4$ & 38 & 27387507 & 27494334 & 173.89 & $7.20 E-04$ & rs11029994 & $9.10 E-04$ \\
\hline 12 & GLIPR1 & 7 & 75874512 & 75895716 & 44.38 & $7.50 E-04$ & rs11180546 & $1.40 E-04$ \\
\hline 18 & FLJ44087 & 44 & 43018148 & 43087001 & 211.76 & $8.40 E-04$ & rs72912678 & $5.30 E-05$ \\
\hline 18 & LINC-ROR & 8 & 54721803 & 54739350 & 45.04 & $8.60 E-04$ & rs1942348 & $8.10 E-04$ \\
\hline
\end{tabular}

TABle 4: Top 20 KEGG, Reactome, and Biocarta $(\mathrm{emp}-\mathrm{P}<0.05)$ pathway results for $\mathrm{BEHL}_{0.5}$ in the typed GWAS data.

\begin{tabular}{lcccc}
\hline Pathway & Chisq- $P$ & Emp- $P$ & Log (chisqP) & Log (empP) \\
\hline KEGG_PHOSPHATIDYLINOSITOL_SIGNALING_SYSTEM & $8.42 E-05$ & $6.60 E-06$ & 4.07483 & 5.18046 \\
REACTOME_PHOSPHOLIPID_METABOLISM & $4.90 E-04$ & $1.86 E-04$ & 3.30987 & 3.73049 \\
REACTOME_DIABETES_PATHWAYS & $4.59 E-04$ & $6.40 E-04$ & 3.33838 & 3.19382 \\
REACTOME_REGULATION_OF_ORNITHINE_DECARBOXYLASE_ODC & $2.49 E-03$ & $1.53 E-03$ & 2.60460 & 2.81531 \\
REACTOME_UNWINDING_OFDNA & $3.69 E-03$ & $1.75 E-03$ & 2.43340 & 2.75696 \\
REACTOME_SYNTHESIS_OF_DNA & $3.69 E-03$ & $1.98 E-03$ & 2.43340 & 2.70333 \\
KEGG_ARACHIDONIC_ACID_METABOLISM & $1.56 E-03$ & $2.04 E-03$ & 2.80687 & 2.69037 \\
REACTOME_DNA_STRAND_ELONGATION & $3.69 E-03$ & $2.23 E-03$ & 2.43340 & 2.65170 \\
BIOCARTA_EDG1_PATHWAY & $2.35 E-03$ & $2.50 E-03$ & 2.62907 & 2.60206 \\
REACTOME_REGULATION_OF_GENE_EXPRESSION_IN_BETA_CELLS & $2.53 E-03$ & $2.56 E-03$ & 2.59605 & 2.59176 \\
REACTOME_METABOLISM_OF_POLYAMINES & $2.78 E-03$ & $2.61 E-03$ & 2.55570 & 2.58336 \\
KEGG_MATURITY_ONSET_DIABETES_OF_THE_YOUNG & $2.53 E-03$ & $2.65 E-03$ & 2.59605 & 2.57675 \\
BIOCARTA_EIF_PATHWAY & $3.68 E-03$ & $2.66 E-03$ & 2.43389 & 2.57512 \\
BIOCARTA_CD40_PATHWAY & $8.21 E-03$ & $2.85 E-03$ & 2.08554 & 2.54516 \\
KEGG_RIG_I_LIKE_RECEPTOR_SIGNALING_PATHWAY & $8.21 E-03$ & $3.04 E-03$ & 2.08554 & 2.51713 \\
REACTOME_RIG_I_MDA5_MEDIATED_INDUCTION_OF_IFN_ALPHA_BETA_- & $8.21 E-03$ & $3.14 E-03$ & 2.08554 & 2.50307 \\
PATHWAYS & $1.86 E-03$ & $3.19 E-03$ & 2.73138 & 2.49621 \\
REACTOME_DESTABILIZATION_OF_MRNA_BY_KSRP & $7.77 E-03$ & $3.19 E-03$ & 2.10930 & 2.49621 \\
REACTOME_EFFECTS_OF_PIP2_HYDROLYSIS & $3.22 E-03$ & $3.20 E-03$ & 2.49276 & 2.49485 \\
REACTOME_AMINE_COMPOUND_SLC_TRANSPORTERS & $7.46 E-03$ & $3.21 E-03$ & 2.12734 & 2.49349 \\
BIOCARTA_AT1R_PATHWAY & & &
\end{tabular}

These results were compared with significant ARHIassociated genes previously reported by other GWAS. Two replicable genes were identified, namely, ACAN [14] for
BEHL at $4.0 \mathrm{kHz}\left(P=4.90 \times 10^{-2}\right)$ and CMIP [32] for BEHL at $8.0 \mathrm{kHz} \quad\left(P=1.30 \times 10^{-2}\right)$. Although the well-known GRM7 gene $[12,13]$ was also identified for BEHL at 
$8.0 \mathrm{kHz}$ and PTA, the $P$ values did not reach the nominal significance level.

3.4. Pathway Enrichment Analysis. The top 20 pathways for $\mathrm{BEHL}_{0.5}$ were sorted according to their empirical $P$ values in Table $4 \mathrm{BEHL}_{1.0}$ : Additional file 9, $\mathrm{BEHL}_{2.0}$ : Additional file $10, \mathrm{BEHL}_{4.0}$ : Additional file 11, $\mathrm{BEHL}_{8.0}$ : Additional file 12, PTA: Additional file 13). For BEHLs at 0.5, 1.0, and $2.0 \mathrm{kHz}$, the main enriched pathways were phosphatidylinositol signaling system; regulation of ornithine decarboxylase; EIF pathway; amine compound SLC transporters; synthesis of PIPS at the plasma membrane; PI metabolism; O glycan biosynthesis; transport of glucose and other sugars; and transport of bile salts and organic acids, metal ions, and amine compounds. By comparison, the main enriched pathways for BEHLs at 4.0 and $8.0 \mathrm{kHz}$ were cysteine and methionine metabolism and adherens junction.

\section{Discussion}

We explored the specific genetic variants in $131 \mathrm{DZ}$ twin pairs that underlie ARHI. VEGAS2 analysis suggested that several genes were nominally associated with BEHLs and PTA. Five consistent genes, namely, C20orf196, GALNT9, INPP4B, SEMA7A, and ARID3B, were observed for BEHLs at $0.5,1.0$, and $2.0 \mathrm{kHz}$. The SEMA7A gene encodes a member of the semaphorin family of proteins that have been found in activated lymphocytes and erythrocytes and which may play a crucial role in immunomodulatory and neuronal processes [33]. Although their functions in ARHI are uncertain, the other genes can also serve as latent candidates for future work. Our comparison of the ARHI-related genes found herein with those reported by previous GWAS obtained two replicable genes, namely, ACAN [14] for BEHL at $4.0 \mathrm{kHz}$ and CMIP [32] for BEHL at $8.0 \mathrm{kHz}$. Using the Shared Harvard Inner Ear Database, Hoffman et al. found that ACAN is expressed in the auditory tissues of mouse [14]. In several developmental phases of mouse, it is mainly expressed in the cochlea and cysts, inner and outer hair cells of the cochlea, and spiral and vestibular ganglia [34-36]. By comparison, CMIP is expressed in the inner ear. Furthermore, Girotto et al. found that this gene is associated with hearing ability at $0.25,1.0$, and $2.0 \mathrm{kHz}$ [32]. In contrast to our findings, a GWAS meta-analysis of ARHI using pure tone audiometry from multiple cohorts reported seven completely different associated loci. This may be explained by the different ethnic and genetic background [37].

Among the enriched ARHI-related pathways, amine compound SLC transporters [38]; phosphatidylinositol signaling system [39]; synthesis of PIPS at the plasma membrane [39-42]; transport of glucose and other sugars bile salts and organic acids metal ions and amine compounds [43-46]; cysteine and methionine metabolism [47, 48]; and adherens junction [49] have been previously reported to be associated with ARHI. Aside from these pathways, other pathways that may be related to ARHI were found, including EIF pathway, PI metabolism, O glycan biosynthesis, and regulation of ornithine decarboxylase. To the best of our knowl- edge, regulation of ornithine decarboxylase had not been reported as associated with ARHI. Ornithine decarboxylase is a key enzyme in the process of polyamine anabolism in the human body. Polyamines have various biological functions, such as antioxidation, free radical scavenging, and intracellular calcium regulation, all of which reportedly have an impact on hearing [50,51]. Accumulating evidence shows that ornithine decarboxylase is associated with disordered cell growth regulation [52-54]. Aside from this pathway, PI metabolism had not been reported to be associated with ARHI. With the discovery of the high expression of the TRPM7 gene in the organ of Corti and cochlea, as well as the detection of TRPM4 immunoreactivity in the inner ear, researchers gradually realized that the TRP channel plays an important role in auditory functions [55-57]. However, TRP channels require PI metabolism to be activated [58]. Therefore, PI metabolism is also closely related to the production of hearing.

We also measured these variants by BEHLs and PTA via GWAS. Several SNPs were found to be suggestively associated with ARHI. We compared these SNPs with significant ARHI-associated SNPs previously reported by other GWAS [12-14, 32, 59]. Nevertheless, we found several promising genetic regions on chromosomes that were nominally associated with ARHI. The association between the genes involved in these promising genetic regions and ARHI could serve as candidates for further research and validation. Furthermore, the enhancer of primary $\mathrm{T}$ helper memory/regulatory cells from the peripheral blood for BEHL at $0.5 \mathrm{kHz}$ was found. Genes involved in immunity and apoptosis are probably related to ARHI $[60,61]$, and the maintenance of systemic immune functions can prevent accelerated ARHI [62]. Hence, T helper memory/regulatory cells may serve as candidate tissues for further investigation of gene expression in animal models.

Investigations into genetically related individuals, such as twins, will enhance genetic association studies, and the use of twin-based designs can efficiently identify both common and rare genetic variants underlying complex traits or diseases. We conducted this GWAS on ARHI in a sample of middle and old-aged Chinese twins, and the utilization of twinbased design will empower genetic association studies and efficiently identify genetic variants underlying ARHI. However, the present GWAS has several limitations. First, owing to the challenges in recruiting and confirming qualified twin participants, we obtained a relatively small sample size. Thus, a GWAS meta-analysis with a larger sample is warranted. In addition, we could not distinguish sensorineural hearing loss from conductive hearing loss because bone conduction test was not performed in this study. Finally, lack of replication of identified signals was performed.

\section{Conclusions}

We identified lists of SNPs reached the suggestive evidence level and found several promising genetic regions on chromosomes associated with ARHI measured by BEHLs and PTA. And sets of genes nominally associated with ARHI were involved in significant biological pathways potentially 
related to pathogenesis of auditory development and hearing impairment. Nevertheless, the potential candidate biomarkers of ARHI reported here should merit further verifications.

\section{Data Availability}

The SNPs datasets for this study have been deposited in the European Variation Archive (EVA) (Accession No. PRJEB23749).

\section{Conflicts of Interest}

The authors declare that they have no competing interests.

\section{Acknowledgments}

We thank Gu Zhu for his help on installing and applicating the related software and performing the statistical analyses. This work was supported by the grants from the National Natural Science Foundation of China (grant numbers 81703292 and 31371024), the China Postdoctoral Science Foundation (2016M590622), the Qingdao Postdoctoral Application Research Project (2015150), the Qingdao Key Health Discipline Development Fund (201909A09), the Qingdao Outstanding Health Professional Development Fund (20190947), and the Shandong Medical and Health Science and Technology Development Program (2015wso329).

\section{Supplementary Materials}

Supplementary 1. Additional file 1: summarised results for chromosome X SNP rs6633657 with trait BEHL $2.0 \mathrm{kHz}$.

Supplementary 2. Additional file 2: query SNP enhancer summary for BEHLs and PTA.

Supplementary 3. Additional file 3: the genes nominally associated with BEHLs at each frequency and PTA from VEGAS2 gene-based analysis $(P<0.05)$.

Supplementary 4. Additional file 4: top 20 genes from VEGAS2 gene-based analysis showing the strongest association with $\mathrm{BEHL}_{1.0}$.

Supplementary 5. Additional file 5: top 20 genes from VEGAS2 gene-based analysis showing the strongest association with $\mathrm{BEHL}_{2.0}$.

Supplementary 6. Additional file 6: top 20 genes from VEGAS2 gene-based analysis showing the strongest association with $\mathrm{BEHL}_{4.0}$.

Supplementary 7. Additional file 7: top 20 genes from VEGAS2 gene-based analysis showing the strongest association with $\mathrm{BEHL}_{8.0}$.

Supplementary 8. Additional file 8: top 20 genes from VEGAS2 gene-based analysis showing the strongest association with PTA.

Supplementary 9. Additional file 9: top $20 \mathrm{KEGG}$, Reactome, and Biocarta pathway results for $\mathrm{BEHL}_{1.0}$ in the typed GWAS data.
Supplementary 10. Additional file 10: top 20 KEGG, Reactome, and Biocarta pathway results for $\mathrm{BEHL}_{2.0}$ in the typed GWAS data.

Supplementary 11. Additional file 11: top 20 KEGG, Reactome, and Biocarta pathway results for $\mathrm{BEHL}_{4.0}$ in the typed GWAS data.

Supplementary 12. Additional file 12: top 20 KEGG, Reactome, and Biocarta pathway results for $\mathrm{BEHL}_{8.0}$ in the typed GWAS data.

Supplementary 13. Additional file 13: top 20 KEGG, Reactome, and Biocarta pathway results for PTA in the typed GWAS data.

\section{References}

[1] C. Liu, X. K. Bu, G. Q. Xing et al., "Epidemiologic study on hearing impairment and ear diseases in old people," Zhonghua Er Bi Yan Hou Tou Jing Wai Ke Za Zhi, vol. 41, no. 9, pp. 661664, 2006.

[2] Taoran Liu, Xia Li, Zengchang Pang, and Shaojie Wang, "Prevalence and influence factors of hearing impairment among rural senior people in Qingdao municipality," Chin Journal of Public Health, vol. 29, no. 8, pp. 1147-1150, 2013.

[3] C. H. Yang, T. Schrepfer, and J. Schacht, "Age-related hearing impairment and the triad of acquired hearing loss," Frontiers in Cellular Neuroscience, vol. 9, 2015.

[4] T. Yamasoba, F. R. Lin, S. Someya, T. Sakamoto, K. Kondo, and A. Kashio, "Current concepts in age-related hearing loss: epidemiology and mechanistic pathways," Hearing Research, vol. 303, pp. 30-38, 2013.

[5] S. K. Momi, L. E. Wolber, S. M. Fabiane, F. M. K. Williams, and A. J. MacGregor, "Genetic and environmental factors in agerelated hearing impairment," Twin Research and Human Genetics, vol. 18, no. 4, pp. 383-392, 2015.

[6] K. Christensen, H. Frederiksen, and H. J. Hoffman, "Genetic and environmental influences on self-reported reduced hearing in the old and oldest old," Journal of the American Geriatrics Society, vol. 49, no. 11, pp. 1512-1517, 2001.

[7] A. Viljanen, P. Era, J. Kaprio, I. Pyykko, T. Rantanen, and M. Koskenvuo, "Genetic and environmental influences on hearing in older women," The Journals of Gerontology Series A: Biological Sciences and Medical Sciences, vol. 62, no. 4, pp. 447-452, 2007.

[8] A. Wingfield, M. Panizzon, M. D. Grant et al., "A twin-study of genetic contributions to hearing acuity in late middle age," The Journals of Gerontology Series A: Biological Sciences and Medical Sciences, vol. 62, no. 11, pp. 1294-1299, 2007.

[9] L. A. Raynor, J. S. Pankow, M. B. Miller et al., "Familial aggregation of age-related hearing loss in an epidemiological study of older adults," American Journal of Audiology, vol. 18, no. 2, pp. 114-118, 2009.

[10] E. Kvestad, N. Czajkowski, N. H. Krog, K. Tambs, and B. Engdahl, "Heritability of hearing loss," Epidemiology, vol. 23, no. 2, pp. 328-331, 2012.

[11] R. Bogo, A. Farah, A. C. Johnson et al., "The role of genetic factors for hearing deterioration across 20 years: a twin study," The Journals of Gerontology Series A: Biological Sciences and Medical Sciences, vol. 70, no. 5, pp. 647-653, 2015. 
[12] L. van Laer, J. R. Huyghe, S. Hannula et al., "A genome-wide association study for age-related hearing impairment in the Saami," European Journal of Human Genetics, vol. 18, no. 6, pp. 685-693, 2010.

[13] R. A. Friedman, L. van Laer, M. J. Huentelman et al., "GRM7 variants confer susceptibility to age-related hearing impairment," Human Molecular Genetics, vol. 18, no. 4, pp. 785796, 2009.

[14] T. J. Hoffmann, B. J. Keats, N. Yoshikawa, C. Schaefer, N. Risch, and L. R. Lustig, "A large genome-wide association study of age-related hearing impairment using electronic health records," Plos Genetics, vol. 12, no. 10, article e1006371, 2016.

[15] Q. Tan, W. Li, and F. Vandin, "Disease-concordant twins empower genetic association studies," Annals of Human Genetics, vol. 81, no. 1, pp. 20-26, 2017.

[16] H. Duan, D. Zhang, Y. Liang et al., "Heritability of age-related hearing loss in middle-aged and elderly Chinese: a populationbased twin study," Ear and Hearing, vol. 40, no. 2, pp. 253259, 2019.

[17] C. Xu, D. Zhang, X. Tian et al., "Genetic and environmental influences on correlations between hearing and cognitive functions in middle and older Chinese twins," Twin Research and Human Genetics, vol. 20, no. 5, pp. 374-379, 2017.

[18] C. Xu, D. Zhang, X. Tian et al., "Genetic and environmental basis in phenotype correlation between physical function and cognition in aging Chinese twins," Twin Research and Human Genetics, vol. 20, no. 1, pp. 60-65, 2017.

[19] A. Becker, A. Busjahn, H. D. Faulhaber et al., "Twin zygosity. Automated determination with microsatellites," Journal of Reproductive Medicine, vol. 42, no. 5, pp. 260-266, 1997.

[20] C. S. Tomsey, M. Kurtz, F. Kist, P. Call, and M. Hockensmith, "Comparison of PowerPlex 16, PowerPlex1.1/2.1, and ABI AmpfISTR profiler plus/COfiler for forensic use," Croatian Medical Journal, vol. 42, no. 3, pp. 239-243, 2001.

[21] R. W. Jackson, H. Snieder, H. Davis, and F. A. Treiber, "Determination of twin zygosity: a comparison of DNA with various questionnaire indices," Twin Research, vol. 4, no. 1, pp. 12-18, 2001.

[22] X. Zhou and M. Stephens, "Genome-wide efficient mixedmodel analysis for association studies," Nature Genetics, vol. 44, no. 7, pp. 821-824, 2012.

[23] F. Dudbridge and A. Gusnanto, "Estimation of significance thresholds for genomewide association scans," Genetic Epidemiology, vol. 32, no. 3, pp. 227-234, 2008.

[24] A. Loukola, J. Wedenoja, K. Keskitalo-Vuokko et al., "Genome-wide association study on detailed profiles of smoking behavior and nicotine dependence in a twin sample," Molecular Psychiatry, vol. 19, no. 5, pp. 615-624, 2014.

[25] L. D. Ward and M. Kellis, "HaploReg: a resource for exploring chromatin states, conservation, and regulatory motif alterations within sets of genetically linked variants," Nucleic Acids Research, vol. 40, no. D1, pp. D930-D934, 2012.

[26] L. D. Ward and M. Kellis, "HaploReg v4: systematic mining of putative causal variants, cell types, regulators and target genes for human complex traits and disease," Nucleic Acids Research, vol. 44, no. D1, pp. D877-D881, 2016.

[27] J. Z. Liu, A. F. McRae, D. R. Nyholt et al., "A versatile genebased test for genome-wide association studies," American Journal of Human Genetics, vol. 87, no. 1, pp. 139-145, 2010.
[28] A. Mishra and S. Macgregor, "VEGAS2: software for more flexible gene-based testing," Twin Research and Human Genetics, vol. 18, no. 1, pp. 86-91, 2015.

[29] C. Xu, D. Zhang, Y. Wu et al., "A genome-wide association study of cognitive function in Chinese adult twins," Biogerontology, vol. 18, no. 5, pp. 811-819, 2017.

[30] D. Lamparter, D. Marbach, R. Rueedi, Z. Kutalik, and S. Bergmann, "Fast and rigorous computation of gene and pathway scores from SNP-based summary statistics," Plos Computational Biology, vol. 12, no. 1, article e1004714, 2016.

[31] Q. T. Ostrom, W. Coleman, W. Huang et al., "Sex-specific gene and pathway modeling of inherited glioma risk," Neuro-Oncology, vol. 21, no. 1, pp. 71-82, 2019.

[32] G. Girotto, N. Pirastu, R. Sorice et al., "Hearing function and thresholds: a genome-wide association study in European isolated populations identifies new loci and pathways," Journal of Medical Genetics, vol. 48, no. 6, pp. 369-374, 2011.

[33] R. Garcia-Areas, S. Libreros, and V. Iragavarapu-Charyulu, "Semaphorin7A: branching beyond axonal guidance and into immunity," Immunologic Research, vol. 57, no. 1-3, pp. 8185, 2013.

[34] D. I. Scheffer, J. Shen, D. P. Corey, and Z. Y. Chen, "Gene expression by mouse inner ear hair cells during development," Journal of Neuroscience, vol. 35, no. 16, pp. 6366-6380, 2015.

[35] J. B. Shin, J. F. Krey, A. Hassan et al., "Molecular architecture of the chick vestibular hair bundle," Nature Neuroscience, vol. 16, no. 3, pp. 365-374, 2013.

[36] H. Liu, J. L. Pecka, Q. Zhang, K. W. Beisel, D. Z. Z. He, and G. A. Soukup, "Characterization of transcriptomes of cochlear inner and outer hair cells," Journal of Neuroscience, vol. 34, no. 33, pp. 11085-11095, 2014.

[37] A. P. Nagtegaal, L. Broer, N. R. Zilhao et al., "Genome-wide association meta-analysis identifies five novel loci for agerelated hearing impairment," Scientific Reports, vol. 9, no. 1, 2019.

[38] L. Schweitzer, J. H. Casseday, A. Sjoerdsma, P. P. McCann, and J. V. Bartolome, "Identification of polyamines in the cochlea of the rat and their potential role in hearing," Brain Research Bulletin, vol. 16, no. 2, pp. 215-218, 1986.

[39] X. Chen, X. Zhao, H. Cai et al., "The role of sodium hydrosulfide in attenuating the aging process via PI3K/AKT and CaMKK $\beta /$ AMPK pathways," Redox Biology, vol. 12, pp. 987-1003, 2017.

[40] J. Nigou, L. G. Dover, and G. S. Besra, "Purification and biochemical characterization of Mycobacterium tuberculosis SuhB, an inositol monophosphatase involved in inositol biosynthesis," Biochemistry, vol. 41, no. 13, pp. 4392-4398, 2002.

[41] K. Y. Lee, "Pathophysiology of age-related hearing loss (peripheral and central)," Korean Journal of Audiology, vol. 17, no. 2, pp. 45-49, 2013.

[42] K. Kiakojouri, M. Monadi, M. Sheikhzadeh, P. Taghinejad Omran, M. A. Bayani, and S. Khafri, "Investigation of auditory thresholds in type 2 diabetic patients compared to nondiabetic cases," Caspian Journal of Internal Medicine, vol. 5, no. 2, pp. 99-102, 2014.

[43] I. Ramasamy, "Update on the molecular biology of dyslipidemias," Clinica Chimica Acta, vol. 454, pp. 143-185, 2016.

[44] Z. du, Y. Yang, Y. Hu et al., "A long-term high-fat diet increases oxidative stress, mitochondrial damage and apoptosis in the inner ear of d-galactose-induced aging rats," Hearing Research, vol. 287, no. 1-2, pp. 15-24, 2012. 
[45] H. Liu, W. Wang, C. Zhang et al., "Heritability and genomewide association study of plasma cholesterol in Chinese adult twins," Frontiers in Endocrinology, vol. 9, p. 677, 2018.

[46] C. M. Villares, J. S. R. Carbajo, J. D. Calvo, M. F. Pello, P. P. Blanco, and M. T. Risueno, "Lipid profile and hearing-loss aged-related," Nutricion Hospitalaria, vol. 20, no. 1, pp. 52$57,2005$.

[47] S. Someya, W. Yu, W. C. Hallows et al., "Sirt3 mediates reduction of oxidative damage and prevention of age-related hearing loss under caloric restriction," Cell, vol. 143, no. 5, pp. 802$812,2010$.

[48] H. Zhang and H. J. Forman, "Glutathione synthesis and its role in redox signaling," Seminars in Cell and Developmental Biology, vol. 23, no. 7, pp. 722-728, 2012.

[49] X. Shi, "Pathophysiology of the cochlear intrastrial fluid-blood barrier (review)," Hearing Research, vol. 338, pp. 52-63, 2016.

[50] T. R. Anderson and S. M. Schanberg, "Ornithine decarboxylase activity in developing rat brain," Journal of Neurochemistry, vol. 19, no. 6, pp. 1471-1481, 1972.

[51] K. C. Das and H. P. Misra, "Hydroxyl radical scavenging and singlet oxygen quenching properties of polyamines," Molecular and Cellular Biochemistry, vol. 262, no. 1/2, pp. 127-133, 2004.

[52] Y. Xie, C. D. Dong, Q. Wu et al., "Ornithine decarboxylase inhibition downregulates multiple pathways involved in the formation of precancerous lesions of esophageal squamous cell cancer," Molecular Carcinogenesis, vol. 59, no. 2, pp. 215-226, 2020.

[53] R. C. Geck, J. R. Foley, T. Murray Stewart, J. M. Asara, R. A. Casero Jr., and A. Toker, "Inhibition of the polyamine synthesis enzyme ornithine decarboxylase sensitizes triple-negative breast cancer cells to cytotoxic chemotherapy," Journal of Biological Chemistry, vol. 295, no. 19, pp. 6263-6277, 2020.

[54] C. Wu, M. Yang, and H. Chen, "Inhibition effect of miR-150 on the progression of oral squamous cell carcinoma by data analysis model based on independent sample T-test," Saudi Journal of Biological Sciences, vol. 27, no. 2, pp. 599-605, 2020.

[55] M. Sakuraba, J. Murata, R. Teruyama et al., "Spatiotemporal expression of TRPM4 in the mouse cochlea," Journal of Neuroscience Research, vol. 92, no. 10, pp. 1409-1418, 2014.

[56] M. P. Cuajungco, C. Grimm, and S. Heller, "TRP channels as candidates for hearing and balance abnormalities in vertebrates," Biochimica et Biophysica Acta, vol. 1772, no. 8, pp. 1022-1027, 2007.

[57] Y. Asai, J. R. Holt, and G. S. G. Géléoc, “A quantitative analysis of the spatiotemporal pattern of transient receptor potential gene expression in the developing mouse cochlea," Journal of the Association for Research in Otolaryngology, vol. 11, no. 1, pp. 27-37, 2010.

[58] M. Gees, B. Colsoul, and B. Nilius, "The role of transient receptor potential cation channels in Ca2+ signaling," Cold Spring Harbor Perspectives in Biology, vol. 2, no. 10, 2010.

[59] E. Fransen, S. Bonneux, J. J. Corneveaux et al., "Genome-wide association analysis demonstrates the highly polygenic character of age-related hearing impairment," European Journal of Human Genetics, vol. 23, no. 1, pp. 110-115, 2015.

[60] H. Iwai, S. Lee, M. Inaba et al., "Correlation between accelerated presbycusis and decreased immune functions," Experimental Gerontology, vol. 38, no. 3, pp. 319-325, 2003.
[61] Y. Dong, M. Li, P. Liu, Y. Zhao, and J. Shi, "Genes involved in immunity and apoptosis are associated with human presbycusis based on microarray analysis," Acta Oto-Laryngologica, vol. 134, no. 6, pp. 601-608, 2014.

[62] H. Iwai, S. Baba, M. Omae, T. Yamashita, S. Ikehara, and S. Lee, "Maintenance of systemic immune functions prevents accelerated presbycusis," Brain Research, vol. 1208, pp. 8-16, 2008. 